\title{
Attitude Stabilization Control of a Quadrotor UAV by Using Backstepping Approach
}

\author{
Xing Huo, ${ }^{1}$ Mingyi Huo, ${ }^{2}$ and Hamid Reza Karimi ${ }^{3}$ \\ ${ }^{1}$ College of Engineering, Bohai University, Jinzhou 121000, China \\ ${ }^{2}$ Department of Control Science and Engineering, Harbin Institute of Technology, Harbin 150001, China \\ ${ }^{3}$ Department of Engineering, Faculty of Engineering and Science, The University of Agder, N-4898 Grimstad, Norway
}

Correspondence should be addressed to Xing Huo; hmyi888@163.com

Received 29 December 2013; Accepted 13 January 2014; Published 19 February 2014

Academic Editor: Xudong Zhao

Copyright (C) 2014 Xing Huo et al. This is an open access article distributed under the Creative Commons Attribution License, which permits unrestricted use, distribution, and reproduction in any medium, provided the original work is properly cited.

\begin{abstract}
The modeling and attitude stabilization control problems of a four-rotor vertical takeoff and landing unmanned air vehicle (UAV) known as the quadrotor are investigated. The quadrotor's attitude is represented by the unit quaternion rather than Euler angles to avoid singularity problem. Taking dynamical behavior of motors into consideration and ignoring aerodynamic effect, a nonlinear controller is developed to stabilize the attitude. The control design is accomplished by using backstepping control technique. The proposed control law is based on the compensation for the Coriolis and gyroscope torques. Applying Lyapunov stability analysis proves that the closed-loop attitude system is asymptotic stable. Moreover, the controller can guarantee that all the states of the system are uniformly ultimately bounded in the presence of external disturbance torque. The effectiveness of the proposed control approach is analytically authenticated and also validated via simulation study.
\end{abstract}

\section{Introduction}

In the past decade, the small UAV market has grown rapidly. Small UAVs are applied in various areas such as surveillance, reconnaissance, and aerial photography. In particular, quadrotor helicopters are an emerging rotorcraft concept for UAV platforms. The particular interest of the research community in the quadrotor design can be linked to two main advantages over comparable vertical takeoff and landing (VTOL) UAVs, such as helicopters. First, quadrotors do not require complex mechanical control linkages for rotor actuation, relying instead on fixed pitch rotors and using variation in motor speed for vehicle control. This simplifies both the design and maintenance of the vehicle. Second, the use of four rotors ensures that individual rotors are smaller in diameter than the equivalent main rotor on a helicopter, relative to the airframe size. Consequently, the last decade has seen many successfully developed platforms of micro-UAVs, especially the quadrotor UAV, such as Australia National University's X-4 flyer aircraft prototype [1], the Stanford University's STARMAC quadrotor [2], the Swiss Federal Institute's OS4 aircraft prototype [3], and the University of Pennsylvania's Grasp micro-UAV test bed [4].

To guide a quadrotor to accomplish the planned mission, a control system needs to be designed. It consists of position control for translational motion and attitude control for rotation motion. In this study, the design of controller for attitude control is investigated. One distinct feature of attitude dynamics of a quadrotor is that its configuration manifold is not linear; it evolves on a nonlinear manifold, referred to as the special orthogonal group. This yields important and unique properties that cannot be observed from dynamic systems evolving on a linear space. As a result, the attitude control problem of a rigid body has been investigated by several researchers, and a wide class of controllers based on classic or modern control theory has been proposed. For instance, based on the conventional proportional-integer-derivative (PID) approach, an model-independent PD controller by using quaternion-based feedback was proposed to stabilize the quadrotor attitude system [5]. In [6], the design of a PID control algorithm for the quadrotor attitude system was presented. The model of the vehicle was modified to simplify 
the controller design. Considering faults occurring on the mounted motors, a control strategy by using gain scheduling incorporating PID was developed in [7]. It successfully achieved attitude and position control even in the presence of actuator faults. In another recent work [8], the problem of stabilization and disturbance rejection of attitude subsystem of a quadrotor was addressed. The controller was designed in the framework of PID control scheme; similar control methods also can be found in [9-13].

In addition to the above PID-based attitude control design, many modern control approaches have also been applied to quadrotor attitude controller design. In [14], an attitude control law was proposed for triple tilting rotor miniUAV. It was robust with respect to dynamical couplings and adverse torques. The work in [15] investigated the application of model reference adaptive control for quadrotor even in the presence of actuator uncertainties. An adaptive law and control laws based on adaptive technique were developed and applied for quadrotor UAV in case of actuator partial loss of effectiveness faults [16]. In [17], the problem of fault tolerant control for quadrotor was addressed by using backstepping control approach. In [18], the output-feedback control system design for a quadrotor was discussed. The position and attitude control was designed by using model reference control and nonlinear control allocation techniques. The position control of vertical take-off and landing UAV without linear velocity measurements was also investigated in [19]. In [20], an adaptive nonlinear attitude stabilization control scheme was synthesized for a quadrotor. The problem of parametric uncertainties in the quadrotor model was investigated, and the controller was designed based on model reference adaptive control technique. In [21], an attitude free position control design was proposed for a quadrotor by using dynamic inversion. In another related study [22], an adaptive law was designed to asymptotically follow an attitude command without the knowledge of the inertia matrix. The proposed control was verified by using a quadrotor UAV model. In [23], a quaternion-based feedback was developed for the attitude stabilization of quadrotor. The control design took into account a priori input bound and is based on nested saturation approach. It forced the closed-loop trajectories to enter in some a priori fixed neighborhood of the origin in a finite time and remain thereafter.

The sliding mode control (SMC) is a powerful theory for controlling uncertain systems [24]. The main advantages are that the SMC system has great robustness with respect to uncertain parameters and external disturbances. Hence, applying SMC to design attitude control for quadrotor has been intensively carried out in recent years. In [25] integral SMC and reinforcement learning control were presented as two design techniques for accommodating the nonlinear disturbances of an outdoor quadrotor. In [26], SMC was used to control a quadrotor UAV in the presence of disturbance and actuator fault. The proposed approach was able to achieve disturbance rejection in the fault-free condition and also able to recover some of performances when a fault occurred. To regulate the attitude angle of a quadrotor to the desired signals, an SMC-based attitude controller was proposed in [27]. In that approach, an adaptive estimator was incorporated, and the adaptive technique was used to estimate the upper bound of the virtual command. The work in [28] explained the developments of the use of SMC for a fully actuated subsystem of a quadrotor to obtain attitude control stability. In [29], an SMC attitude controller was developed for a quadrotor. It allowed for a continuous control robust to external disturbance and model uncertainties to be computed without the use of high control gain. In another work [30], an augmented SMC-based fault-tolerant control was designed theoretically, implemented practically, and tested experimentally in a quadrotor. The problems of propeller damage and actuator fault conditions for tracking control were discussed. Moreover, many other nonlinear control techniques based controller design were also proposed for quadrotor, as suggested in [31-33].

Based on the results available in the literature, this work will investigate the attitude control design of a quadrotor UAV. The attitude orientation of the quadrotor is represented by using unit quaternion. Backstepping technique is adopted to develop the controller. It is shown that the controller can asymptotically stabilize the attitude system. Explicitly taking external disturbance torque acting on the quadrotor into consideration, the controller is able to guarantee the uniformly ultimately bounded stability of the attitude system. All the states of the closed-loop attitude system are governed to be uniformly ultimately bounded. The remainder of this paper is organized as follows. In Section 2, mathematical model of a quadrotor UAV attitude system and problem statement are summarized. A backstepping-based attitude control approach is presented in Section 3, and also the stability of closed-loop system is provided. In Section 4, simulation results with the application of the designed control scheme to a quadrotor are presented. Section 5 presents some concluding remarks and future work.

\section{Mathematical Model and Control Problem Statement}

2.1. Attitude Dynamic Model of a Quadrotor. The quadrotor UAV under consideration consists of a rigid cross frame equipped with four rotors as shown in Figure 1. Those four rotors are divided into front $\left(M_{2}\right)$, back $\left(M_{4}\right)$, left $\left(M_{1}\right)$, and right $\left(M_{3}\right)$ motors. Motors $M_{2}$ and $M_{4}$ rotate in counterclockwise direction, while the other two in clockwise direction. All of the movements can be controlled by the changes of each rotor speed. If a yaw motion is desired, one has to reduce the thrust of one set of rotors and increase the thrust of the other set while maintaining the same total thrust to avoid an up-down motion. Hence, the yaw motion is then realized in the direction of the induced reactive torque. To accomplish roll motion, it should decrease (increase) the speed of motor $M_{3}$ and increase (decrease) the speed of motor $M_{1}$. On the other hand, pitch motion can be maneuvered by decreasing (increasing) the speed of motor $M_{1}$ while increasing (decreasing) the speed of motor $M_{4}$.

Let $\mathscr{F}_{i}\left(X_{I}, Y_{I}, Z_{I}\right)$ fixed with the Earth denote an inertial frame, and let $\mathscr{F}_{b}\left(X_{B}, Y_{B}, Z_{B}\right)$ denote a frame rigidly attached to the quadrotor body as shown in Figure 1. The vector 


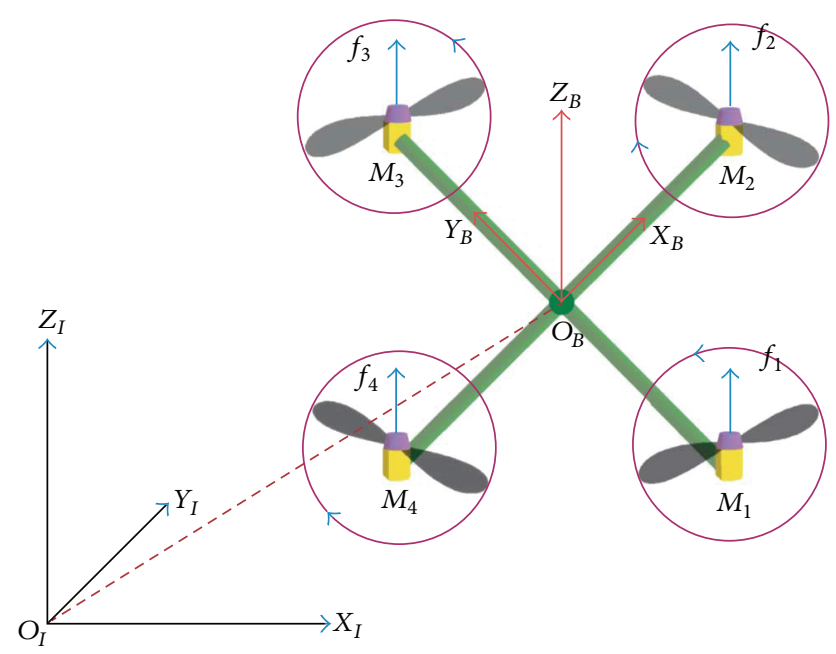

FIGURE 1: The structure of quadrotor UAV and its coordinate reference frames.

$\Theta=\left[\begin{array}{lll}\phi & \theta & \psi\end{array}\right]^{T}$ is the orientation of body frame $\mathscr{F}_{b}$ with respect to $\mathscr{F}_{i}$ called Euler angles. These angles are bounded as follows:

$$
-\frac{\pi}{2}<\phi<\frac{\pi}{2}, \quad-\frac{\pi}{2}<\theta<\frac{\pi}{2}, \quad-\frac{\pi}{2}<\psi<\frac{\pi}{2} .
$$

When using Euler angles to represent quadrotor attitude, direction cosine matrix will be used to describe the kinematic motion of attitude. However, one of the drawbacks with application of the direction cosine matrix is the inherent geometric singularity. It is known that the four-parameter description of the orientation called the quaternion representation can be applied to avoid that drawback. Consequently, the unit quaternion will be used in this work for attitude representation of quadrotor UAV. Ignoring aerodynamic effect, the mathematical model of quadrotor attitude system can be described as follows [5]:

$$
\begin{gathered}
\dot{q}_{0}=-\frac{1}{2} \mathbf{q}^{T} \boldsymbol{\omega} \\
\dot{\mathbf{q}}=\frac{1}{2}\left[q_{0} \mathbf{E}_{3}+\mathbf{S}(\mathbf{q})\right] \boldsymbol{\omega} \\
\mathbf{I} \dot{\boldsymbol{\omega}}=-\mathbf{S}(\boldsymbol{\omega}) \mathbf{I} \boldsymbol{\omega}-\mathbf{G}_{a}+\mathbf{u} \\
I_{m i} \dot{\omega}_{i}=\tau_{i}-f_{i}, \quad i=1,2,3,4,
\end{gathered}
$$

where $\mathbf{Q}=\left[\begin{array}{ll}q_{0} & \mathbf{q}^{T}\end{array}\right]^{T} \in \mathbb{R}^{4}$ with $\mathbf{q} \in \mathbb{R}^{3}$ is the unit quaternion denoting the attitude of quadrotor with respect to the inertial frame $\mathscr{F}_{i}$. It is subject to the equation $q_{0}^{2}+\mathbf{q}^{T} \mathbf{q}=1 . \boldsymbol{\omega} \in \mathbb{R}^{3}$ denotes the angular velocity of the quadrotor with respect to $\mathscr{F}_{i}$ and expressed in $\mathscr{F}_{b} . \mathbf{I} \in \mathbb{R}^{3 \times 3}$ is the positive-definite, symmetric inertia matrix of the UAV. $I_{m} \in \mathbb{R}$ and $\varrho_{i} \in \mathbb{R}$ are the moment of inertia and the speed of the rotor $M_{i}$, respectively. $\mathbf{G}_{a} \in \mathbb{R}^{3}$ denotes the gyroscope torques due to the combination of the rotation of the quadrotor and the four motors; it is given by

$$
\mathbf{G}_{a}=\sum_{i=1}^{4} I_{m i}\left(\boldsymbol{\omega} \times \mathbf{e}_{I}\right)(-1)^{i+1}{\omega_{i}}
$$

and $\mathbf{e}_{I}=\left[\begin{array}{lll}0 & 0 & 1\end{array}\right]^{T}$ denotes the unit vector in $\mathscr{F}_{i}$. The matrix $\mathbf{S}(\mathbf{x})$ is a skew-symmetric matrix such that $\mathbf{S}(\mathbf{x}) \mathbf{y}=\mathbf{x} \times \mathbf{y}$ for any vector for $\mathbf{x}, \mathbf{y} \in \mathbb{R}^{3}$, where " $x$ " denotes the vector crossproduct. $\mathbf{E}_{3}$ denotes the $3 \times 3$ identity matrix.

In (4), $\mathbf{u}=\left[\begin{array}{lll}u_{1} & u_{2} & u_{3}\end{array}\right]^{T} \in \mathbb{R}^{3}$ is the total torque acting on the UAV, and it is generated by four motors.

Consider the following:

$$
\mathbf{u}=\left[\begin{array}{c}
d b\left(\omega_{3}^{2}-\omega_{1}^{2}\right) \\
d b\left(\omega_{2}^{2}-\omega_{4}^{2}\right) \\
\kappa\left(\omega_{2}^{2}+\omega_{4}^{2}-\omega_{1}^{2}-\omega_{3}^{2}\right)
\end{array}\right],
$$

where $d \in \mathbb{R}$ is the distance from the rotors to the center of mass of the quadrotor and $b \in \mathbb{R}$ and $\kappa \in \mathbb{R}$ are two positive parameters depending on the density of air, the radius of the propeller, the number of blades and the geometry, lift, and drag coefficients of the blades [34]. The reaction torque $f_{i} \in \mathbb{R}$ in (5) generated in free air by the motor $M_{i}$ due to motor drag is given by

$$
f_{i}=\kappa \omega_{i}^{2}
$$

Moreover, the four-control input of the attitude system is $\tau_{i}$, $i=1,2,3,4$, which denotes the torques produced by the rotors.

2.2. Problem Statement. Given any initial attitude $\mathbf{Q}$ and angular velocity $\omega$, the control objective to be achieved can be stated as: consider the quadrotor UAV attitude dynamics described by (2) and (5); design an control law $\tau_{i}$ for the motor $M_{i}, i=1,2,3,4$, to stabilize the resulting closed-loop attitude system; that is,

$$
\lim _{t \rightarrow \infty} \mathbf{Q}(t)=\left[\begin{array}{llll}
1 & 0 & 0 & 0
\end{array}\right]^{T}, \quad \lim _{t \rightarrow \infty} \boldsymbol{\omega}(t)=\left[\begin{array}{lll}
0 & 0 & 0
\end{array}\right]^{T} .
$$

\section{Attitude Stabilization Control Design}

Define new state variables as $\mathbf{x}_{1}=\left[1-\left|q_{0}\right| \mathbf{q}^{T}\right]^{T}, \mathbf{x}_{2}=\boldsymbol{\omega}$, and $\mathbf{x}_{3}=\left[\begin{array}{lll}\omega_{1}^{2} & \omega_{2}^{2} & \omega_{3}^{2}\end{array}\right]^{T}$. Then, the attitude system equations (2)(5) can be rewritten as follows:

$$
\begin{gathered}
\dot{\mathbf{x}}_{1}=\left[\begin{array}{lll}
-\operatorname{sgn}\left(q_{0}\right) \dot{q}_{0} & \dot{\mathbf{q}}^{T}
\end{array}\right]^{T} \\
\mathbf{I}_{2}=-\mathbf{S}\left(\mathbf{x}_{2}\right) \mathbf{I} \mathbf{x}_{2}-\mathbf{G}_{a}+\mathbf{u} \\
\mathbf{I}_{m} \dot{\mathbf{x}}_{3}=\mathbf{X}(\boldsymbol{\tau}-\mathbf{f}),
\end{gathered}
$$

where $\mathbf{I}_{m}=\operatorname{diag}\left(I_{m 1}, I_{m 2}, I_{m 3}\right), \mathbf{X}=\operatorname{diag}\left(2 \varrho_{1}, 2 \varrho_{2}, 2 \varrho_{3}\right), \boldsymbol{\tau}=$ $\left[\begin{array}{lll}\tau_{1} & \tau_{2} & \tau_{3}\end{array}\right]^{T}, \mathbf{f}=\left[\begin{array}{lll}f_{1} & f_{2} & f_{3}\end{array}\right]^{T}$, and $\operatorname{sgn}(y)$ is defined as the nonzero sigum function as follows:

$$
\operatorname{sgn}(y)= \begin{cases}-1, & y<0 \\ 1, & y \geq 0 .\end{cases}
$$

For the transformed system equations (10)-(12), backstepping control technique will be applied in this section to 


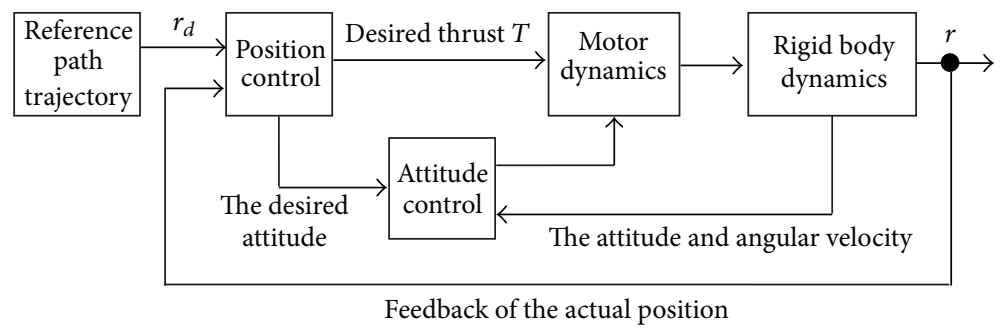

FIgURE 2: The control structure of the quadrotor UAV.

design attitude controller, and the following change of coordinates will be firstly introduced:

$$
\begin{gathered}
\mathbf{z}_{1}=\mathbf{x}_{1} \\
\mathbf{z}_{2}=\mathbf{x}_{2}-\boldsymbol{\alpha}_{1} \\
\mathbf{z}_{3}=\mathbf{x}_{3}-\boldsymbol{\alpha}_{2},
\end{gathered}
$$

where $\boldsymbol{\alpha}_{1} \in \mathfrak{R}^{3}$ and $\boldsymbol{\alpha}_{2} \in \mathfrak{R}^{N}$ are virtual control inputs to be designed later.

Based on the preceding coordinates changes, the following procedures can be followed to design a controller to stabilize the attitude of the considered quadrotor UAV.

Step 1. We start with (14) by considering $\mathbf{x}_{2}$ as the control variable. It is obtained from (2), (3), and (10) that

$$
\begin{aligned}
\dot{\mathbf{z}}_{1} & =\frac{1}{2}\left[\operatorname{sgn}\left(q_{0}\right) \mathbf{q}^{T} \boldsymbol{\omega}\left[q_{0} \mathbf{E}_{3}+\mathbf{S}(\mathbf{q})\right]^{T} \boldsymbol{\omega}^{T}\right]^{T} \\
& =\frac{1}{2}\left[\begin{array}{c}
\operatorname{sgn}\left(q_{0}\right) \mathbf{q}^{T} \\
q_{0} \mathbf{E}_{3}+\mathbf{S}(\mathbf{q})
\end{array}\right] \boldsymbol{\omega} \\
& =\mathbf{P}(\mathbf{Q}) \boldsymbol{\omega}
\end{aligned}
$$

where $\mathbf{P}(\mathbf{Q})=(1 / 2)\left[\begin{array}{c}\operatorname{sgn}\left(q_{0}\right) \mathbf{q}^{T} \\ q_{0} \mathbf{E}_{3}+\mathbf{S}(\mathbf{q})\end{array}\right]$.

The task in this step is to design a virtual control law $\boldsymbol{\alpha}_{1}$ to guarantee $\lim _{t \rightarrow \infty} \mathbf{z}_{1}(t)=\mathbf{0}$. Choose a Lyapunov candidate function as $V_{1}=(1 / 2) \mathbf{z}_{1}^{T} \mathbf{z}_{1}$, and design the virtual control $\boldsymbol{\alpha}_{1}$ as $\boldsymbol{\alpha}_{1}=-l_{1} \mathbf{P}^{T}(\mathbf{Q}) \mathbf{z}_{1}$, where $l_{1} \in \mathbb{R}$ is a positive scalar. Then, applying (15) and (17) yields

$$
\begin{aligned}
\dot{V}_{1} & =\mathbf{z}_{1}^{T} \mathbf{P}(\mathbf{Q}) \boldsymbol{\omega} \\
& =\mathbf{z}_{1}^{T} \mathbf{P}(\mathbf{Q})\left(\mathbf{z}_{2}-l_{1} \mathbf{P}^{T}(\mathbf{Q}) z_{1}\right) \\
& =-l_{1} \mathbf{z}_{1}^{T} \mathbf{P}(\mathbf{Q}) \mathbf{P}^{T}(\mathbf{Q}) \mathbf{z}_{1}+\mathbf{z}_{1}^{T} \mathbf{P}(\mathbf{Q}) \mathbf{z}_{2} .
\end{aligned}
$$

Hence, if $\mathbf{z}_{2}=\mathbf{0}$, then $\dot{V}_{1}=-l_{1} \mathbf{z}_{1}^{T} \mathbf{P}(\mathbf{Q}) \mathbf{P}^{T}(\mathbf{Q}) \mathbf{z}_{1}=$ $-l_{1}\left\|\mathbf{P}^{T}(\mathbf{Q}) \mathbf{z}_{1}\right\|^{2}<0$ for all $\mathbf{P}^{T}(\mathbf{Q}) \mathbf{z}_{1} \neq \mathbf{0}$. By using Lyapunov stability theory [35], it can prove that

$$
\lim _{t \rightarrow \infty}\left\|\mathbf{P}^{T}(\mathbf{Q}) \mathbf{z}_{1}\right\|=\mathbf{0}
$$

On the other hand, it can be obtained from the definition of $\mathbf{P}(\mathbf{Q})$ that

$$
\begin{aligned}
\mathbf{P}^{T}(\mathbf{Q}) \mathbf{z}_{1} & =\frac{1}{2}\left[\begin{array}{c}
\operatorname{sgn}\left(q_{0}\right) \mathbf{q}^{T} \\
q_{0} \mathbf{E}_{3}+\mathbf{S}(\mathbf{q})
\end{array}\right]^{T}\left[1-\left|q_{0}\right| \mathbf{q}^{T}\right]^{T} \\
& =\frac{1}{2} \operatorname{sgn}\left(q_{0}\right) \mathbf{q} .
\end{aligned}
$$

Hence, it leaves (19)-(20) as $\lim _{t \rightarrow \infty} \operatorname{sgn}\left(q_{0}\right) \mathbf{q}=\mathbf{0}$. Then, using (13) leads to $\lim _{t \rightarrow \infty} \mathbf{q}=\mathbf{0}$.

The quadrotor UAV actually is an underactuated system because it has six degrees of freedom while it has only four inputs. The collective input (or throttle input) is the sum of the thrusts of each motor. Hence, the control structure of an rigid quadrotor is illustrated in Figure 2. Position control and attitude control are included. Assumed that the desired total thrust supplied by the position control is $T$, which is generated by the four rotors and given by

$$
T=b \sum_{i=1}^{4} \omega_{i}^{2} .
$$

Accordingly, the desired speed of the four motors can be obtained from (7) and (21); that is, $\boldsymbol{\eta}=\mathbf{N x}_{3}$ with $\boldsymbol{\eta}=$ $\left[\begin{array}{llll}u_{1} & u_{2} & u_{3} & T\end{array}\right]^{T}$ and

$$
\mathbf{N}=\left[\begin{array}{cccc}
0 & d b & 0 & -d b \\
d b & 0 & -d b & 0 \\
\kappa & -\kappa & \kappa & -\kappa \\
b & b & b & b
\end{array}\right]
$$

where $\mathbf{N}$ is nonsingular as long as $d b \kappa \neq 0$.

Step 2. From (22), one has $\mathbf{u}=\boldsymbol{\Xi} \mathbf{N x}_{3}$, where

$$
\Xi=\left[\begin{array}{llll}
1 & 0 & 0 & 0 \\
0 & 1 & 0 & 0 \\
0 & 0 & 1 & 0 \\
0 & 0 & 0 & 0
\end{array}\right] .
$$

We now differentiate the second error, $\mathbf{z}_{2}$, using (15) to give

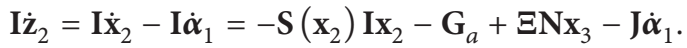

Choose another Lyapunov candidate function $V_{2}=V_{1}+$ $(1 / 2) \mathbf{z}_{2}^{T} \mathbf{I} \mathbf{z}_{2}$, and design the virtual control law $\boldsymbol{\alpha}_{2}$ as

$$
\boldsymbol{\alpha}_{2}=(\boldsymbol{\Xi N})^{\dagger}\left[\mathbf{S}\left(\mathbf{x}_{2}\right) \mathbf{I} \mathbf{x}_{2}-\mathbf{P}^{T}(\mathbf{Q}) \mathbf{z}_{1}-l_{2} \mathbf{z}_{2}+\mathbf{G}_{a}+\mathbf{I} \dot{\boldsymbol{\alpha}}_{1}\right],
$$


where $l_{2} \in \mathbb{R}$ is a positive constant and $(\cdot)^{\dagger}$ denotes the pseudo inverse of a full-row rank matrix.

Differentiating both sides of $V_{2}$ and inserting (24) and (25) yield

$$
\begin{aligned}
\dot{V}_{2}= & \dot{V}_{1}+\mathbf{z}_{2}^{T} \mathbf{I} \dot{\mathbf{z}}_{2} \\
= & -l_{1} \mathbf{z}_{1}^{T} \mathbf{P}(\mathbf{Q}) \mathbf{P}^{T}(\mathbf{Q}) \mathbf{z}_{1}+\mathbf{z}_{1}^{T} \mathbf{P}(\mathbf{Q}) \mathbf{z}_{2} \\
& +\mathbf{z}_{2}^{T}\left[-\mathbf{S}\left(\mathbf{x}_{2}\right) \mathbf{I} \mathbf{x}_{2}-\mathbf{G}_{a}+\mathbf{\Xi N}\left(\mathbf{z}_{3}+\boldsymbol{\alpha}_{2}\right)-\mathbf{I} \dot{\boldsymbol{\alpha}}_{1}\right] \\
= & -l_{1} \mathbf{z}_{1}^{T} \mathbf{P}(\mathbf{Q}) \mathbf{P}^{T}(\mathbf{Q}) \mathbf{z}_{1}-l_{2} \mathbf{z}_{2}^{T} \mathbf{z}_{2}+\mathbf{z}_{2}^{T} \mathbf{\Xi N \mathbf { z } _ { 3 } .}
\end{aligned}
$$

Again, if $\mathbf{z}_{3}=\mathbf{0}$, one has $\dot{V}_{2}=-l_{1}\left\|\mathbf{P}^{T}(\mathbf{Q}) \mathbf{z}_{1}\right\|^{2}-l_{2}\left\|\mathbf{z}_{2}\right\|^{2}$, and thus both $\mathbf{z}_{1}$ and $\mathbf{z}_{2}$ will converge to zero asymptotically.

According to the analysis in Steps 1 and 2, if $\mathbf{z}_{3}$ can be driven to zero by designing an appropriate controller, then $\mathbf{z}_{1}$ and $\mathbf{z}_{2}$ will also be governed to zero, and thus the quadrotor attitude will be stabilized. In the following theorem, we summarize our control solution to drive $\mathbf{z}_{3}$ to zero by incorporating backstepping-based control action.

Theorem 1. Consider the quadrotor attitude system described by (2)-(5); design a nonlinear backstepping controller as follows:

$$
\boldsymbol{\tau}=\mathbf{X}^{-1}\left[-(\boldsymbol{\Xi N})^{T} \mathbf{z}_{2}-l_{3} \mathbf{z}_{3}+\mathbf{I}_{m} \dot{\boldsymbol{\alpha}}_{2}\right]+\mathbf{f},
$$

where $l_{3} \in \mathbb{R}$ is an positive control gain. Then, the closed-loop attitude system is asymptotically stable; that is, $\lim _{t \rightarrow \infty} \mathbf{q}(t)=$ $\left[\begin{array}{lll}0 & 0 & 0\end{array}\right]^{T}, \lim _{t \rightarrow \infty} \boldsymbol{\omega}(t)=\left[\begin{array}{lll}0 & 0 & 0\end{array}\right]^{T}$.

Proof. It can be obtained from (12) and (16) that

$$
\mathbf{I}_{m} \dot{\mathbf{z}}_{3}=\mathbf{I}_{m} \mathbf{x}_{3}-\mathbf{I}_{m} \dot{\boldsymbol{\alpha}}_{2}=\mathbf{X}(\boldsymbol{\tau}-\mathbf{f})-\mathbf{I}_{m} \dot{\boldsymbol{\alpha}}_{2} .
$$

Consider another candidate Lyapunov function $V_{3}$ as follows:

$$
V_{3}=V_{2}+\frac{1}{2} \mathbf{z}_{3}^{T} \mathbf{I}_{m} \mathbf{z}_{3}
$$

Differentiating (29) and inserting (26) and (28) result in

$$
\begin{aligned}
\dot{V}_{3}= & \dot{V}_{2}+\mathbf{z}_{3}^{T} \mathbf{I}_{m} \dot{\mathbf{z}}_{3} \\
= & -l_{1} \mathbf{z}_{1}^{T} \mathbf{P}(\mathbf{Q}) \mathbf{P}^{T}(\mathbf{Q}) \mathbf{z}_{1}-l_{2} \mathbf{z}_{2}^{T} \mathbf{z}_{2} \\
& +\mathbf{z}_{2}^{T} \mathbf{\Xi} \mathbf{N} \mathbf{z}_{3}+\mathbf{z}_{3}^{T}\left[\mathbf{X}(\boldsymbol{\tau}-\mathbf{f})-\mathbf{I}_{m} \dot{\boldsymbol{\alpha}}_{2}\right] .
\end{aligned}
$$

With the developed controller equation (27), (30) can be simplified into the following form:

$$
\dot{V}_{3}=-l_{1} \mathbf{z}_{1}^{T} \mathbf{P}(\mathbf{Q}) \mathbf{P}^{T}(\mathbf{Q}) \mathbf{z}_{1}-l_{2} \mathbf{z}_{2}^{T} \mathbf{z}_{2}-l_{3} \mathbf{z}_{3}^{T} \mathbf{z}_{3} \leq 0 .
$$

Investigating of $V_{3}$ in (29) shows clearly that $V_{3}>0$ for all $\mathbf{z}_{i}$, $i=1,2,3$ and also that $V_{3} \rightarrow \infty$ when $\mathbf{z}_{i} \rightarrow \infty$. Therefore, in accordance with LaSalle-Yoshizawa's theorem [35], $\dot{V}_{3}$ from (31) fulfills

$$
\dot{V}_{3} \leq-W\left(\mathbf{z}_{1}, \mathbf{z}_{2}, \mathbf{z}_{3}\right) \leq 0
$$

where $W\left(\mathbf{z}_{1}, \mathbf{z}_{2}, \mathbf{z}_{3}\right)$ is a continuous function. Then, all solution $\mathbf{z}_{i}(t)$ are uniformly globally bounded and

$$
\lim _{t \rightarrow \infty} W\left(\mathbf{z}_{1}, \mathbf{z}_{2}, \mathbf{z}_{3}\right)=0 .
$$

Hence, the controller is uniformly globally asymptotically stable since additionally $W\left(\mathbf{z}_{1}, \mathbf{z}_{2}, \mathbf{z}_{3}\right)>0$. Moreover, it can be concluded from (31)-(33) that

$$
\lim _{t \rightarrow \infty} \mathbf{z}_{i}(t)=\mathbf{0} \text {. }
$$

To this end, it can be obtained from (14)-(16), the definition of $\boldsymbol{\alpha}_{1}$ and $\boldsymbol{\alpha}_{2}$, and (34) that

$$
\lim _{t \rightarrow \infty} \mathbf{q}(t)=\left[\begin{array}{lll}
0 & 0 & 0
\end{array}\right]^{T}, \quad \lim _{t \rightarrow \infty} \boldsymbol{\omega}(t)=\left[\begin{array}{lll}
0 & 0 & 0
\end{array}\right]^{T} .
$$

Summarizing the above analysis, it can come to the conclusion that the closed-loop attitude system of the considered quadrotor UAV is asymptotically stable. Thereby the proof is completed here.

The result obtained from Theorem 1 can only be guaranteed when the quadrotor UAV is free of external disturbance. However, this case will never be true in practical flying, especially when the quadrotor flies outdoor. Assume that the external disturbance torque acting on the quadrotor is $\mathbf{u}_{d} \in$ $\mathbb{R}^{3}$; then the dynamics of the quadrotor equation (4) or (11) will be changed as follows:

$$
\mathbf{I} \dot{\mathbf{x}}_{2}=-\mathbf{S}\left(\mathbf{x}_{2}\right) \mathbf{I} \mathbf{x}_{2}-\mathbf{G}_{a}+\mathbf{u}+\mathbf{u}_{d} .
$$

Although the disturbance torque $\mathbf{u}_{d}$ is inevitable, it is always bounded in practice. Hence, it is reasonable to assume that there exists a positive scalar $d_{\max } \in \mathbb{R}$ such that $\left\|\mathbf{u}_{d}\right\| \leq$ $d_{\text {max }}$.

Theorem 2. Consider the quadrotor attitude system described by (2)-(5) in the presence of external disturbance torque $\mathbf{u}_{d}$, with application of the controller equation (27); suppose that the control gain $l_{2}$ is chosen to satisfy

$$
l_{2}-\varepsilon>0
$$

where $\varepsilon \in \mathbb{R}$ is a positive scalar specified by the designer. Then, the closed-loop attitude system is uniformly ultimately bounded stable.

Proof. Using the almost the same analysis as in the proof of Theorem 1, one has

$$
\dot{V}_{2}=-l_{1} \mathbf{z}_{1}^{T} \mathbf{P}(\mathbf{Q}) \mathbf{P}^{T}(\mathbf{Q}) \mathbf{z}_{1}-l_{2} \mathbf{z}_{2}^{T} \mathbf{z}_{2}+\mathbf{z}_{2}^{T} \boldsymbol{\Xi} \mathbf{N} \mathbf{z}_{3}+\mathbf{z}_{2}^{T} \mathbf{u}_{d}
$$

Then, it leaves $\dot{V}_{3}$ in (30) as

$$
\dot{V}_{3}=-l_{1} \mathbf{z}_{1}^{T} \mathbf{P}(\mathbf{Q}) \mathbf{P}^{T}(\mathbf{Q}) \mathbf{z}_{1}-l_{2} \mathbf{z}_{2}^{T} \mathbf{z}_{2}-l_{3} \mathbf{z}_{3}^{T} \mathbf{z}_{3}+\mathbf{z}_{2}^{T} \mathbf{u}_{d} .
$$

Applying the Young's inequality, the following inequality can be established:

$$
\mathbf{z}_{2}^{T} \mathbf{u}_{d} \leq \varepsilon\left\|\mathbf{z}_{2}\right\|^{2}+\frac{1}{4 \varepsilon}\left\|\mathbf{u}_{d}\right\|^{2} \leq \varepsilon\left\|\mathbf{z}_{2}\right\|^{2}+\frac{1}{4 \varepsilon} d_{\max }^{2} .
$$


TABLE 1: Quadrotor UAV model parameters.

\begin{tabular}{lccc}
\hline Parameters & Description & Value & Units \\
\hline$g$ & Gravity & 9.81 & $\mathrm{~m} / \mathrm{s}^{2}$ \\
$m$ & Mass & 0.468 & $\mathrm{~kg}$ \\
$d$ & Distance & 0.225 & $\mathrm{~m}$ \\
$I_{m i}, i=1,2,3$ & Rotor inertia & $3.4 \times 10^{-5}$ & $\mathrm{kgm}^{2}$ \\
$I_{x}$ & Roll inertia & $4.9 \times 10^{-3}$ & $\mathrm{kgm}^{2}$ \\
$I_{y}$ & Pitch inertia & $4.9 \times 10^{-3}$ & $\mathrm{kgm}^{2}$ \\
$I_{z}$ & Yaw inertia & $8.8 \times 10^{-3}$ & $\mathrm{kgm}^{2}$ \\
$R_{a}$ & Motor resistance & 0.67 & $\Omega$ \\
$k_{m}$ & Motor constant & $4.3 \times 10^{-3}$ & $\mathrm{Nm} / \mathrm{A}$ \\
$k_{g}$ & Gear ratio & 5.6 & \\
$b$ & Proportionality constant & $2.9 \times 10^{-5}$ & \\
$\kappa$ & Proportionality constant & $1.1 \times 10^{-6}$ & \\
\hline
\end{tabular}

It is thus obtained from (40) that $\dot{V}_{3}$ is bounded by

$$
\begin{aligned}
\dot{V}_{3} & =-l_{1} \mathbf{z}_{1}^{T} \mathbf{P}(\mathbf{Q}) \mathbf{P}^{T}(\mathbf{Q}) \mathbf{z}_{1}-l_{2} \mathbf{z}_{2}^{T} \mathbf{z}_{2}-l_{3} \mathbf{z}_{3}^{T} \mathbf{z}_{3}+\mathbf{z}_{2}^{T} \mathbf{u}_{d} \\
& \leq-l_{1} \mathbf{z}_{1}^{T} \mathbf{P}(\mathbf{Q}) \mathbf{P}^{T}(\mathbf{Q}) \mathbf{z}_{1}-\left(l_{2}-\varepsilon\right) \mathbf{z}_{2}^{T} \mathbf{z}_{2}-l_{3} \mathbf{z}_{3}^{T} \mathbf{z}_{3}+\frac{1}{4 \varepsilon} d_{\max }^{2} .
\end{aligned}
$$

With the choice of control gains in (37), it leads to

$$
\dot{V}_{3} \leq-2 \bar{l} V_{3}+\frac{d_{\max }^{2}}{4 \varepsilon},
$$

where $\bar{l}=\min \left\{l_{1} \lambda_{\min }\left(\mathbf{P}(\mathbf{Q}) \mathbf{P}^{T}(\mathbf{Q})\right),\left(l_{2}-\varepsilon\right) / \lambda_{\max }(\mathbf{I})\right.$, $\left.l_{3} / \lambda_{\max }\left(\mathbf{I}_{m}\right)\right\}, \lambda_{\min }(\cdot)$ and $\lambda_{\max }(\cdot)$ denote the minimum and the maximum eigenvalue of matrix, respectively. Using Theorem 4.18 (page 172) in [35], it can be concluded from (42) that $V_{3}$ is uniformly ultimately bounded together with the states $\mathbf{z}_{i}$, $i=1,2,3$. More precisely, there exists a finite-time $\bar{t}_{0} \in \mathfrak{R}_{+}$ such that $\left\|\mathbf{z}_{i}(t)\right\| \leq \varepsilon_{1}^{*},\left\|\mathbf{z}_{a}(t)\right\| \leq \varepsilon_{1}^{*}$ for any $\varepsilon_{1}^{*}>d_{\max } / 2 \sqrt{\bar{l}_{\varepsilon}}$ and $t \geq \bar{t}_{0}, i=1,2,3$. In other words, the closed-loop attitude system is uniformly ultimately bounded stable. Hence, the proof of Theorem 2 is completed.

\section{Numerical Example}

In this section the properties of the proposed attitude stabilization approach is evaluated and simulated numerically by the quadrotor model presented in [5]. The physical parameters of this model are listed in Table 1 . At time $t=0$, the initial attitude of the quadrotor is $\Theta(0)=\left[\begin{array}{lll}35 & -45 & 18\end{array}\right]^{T} \mathrm{deg}$, corresponding to the unit quaternion values $\mathbf{q}(0)=$ $\left[\begin{array}{lll}0.3315 & -0.3170 & 0.0242\end{array}\right]^{T}$; the initial angular velocity is of $\omega(0)=\left[\begin{array}{lll}0 & 0 & 0\end{array}\right]^{T} \mathrm{rad} / \mathrm{sec}$. The control gains for the controller equation (27) are chosen as $l_{1}=0.02, l_{2}=0.075$, and $l_{3}=$ 0.008 . Moreover, attitude and angular velocity sensor noises $\left(\sigma_{p}^{2}\right): 0.0001(1 \sigma)$ are modeled as zero-mean Gaussian random variables with variance $\sigma_{p}^{2}$.

In the ideal case that the quadrotor is free of external disturbance torques, with application of the controller equation

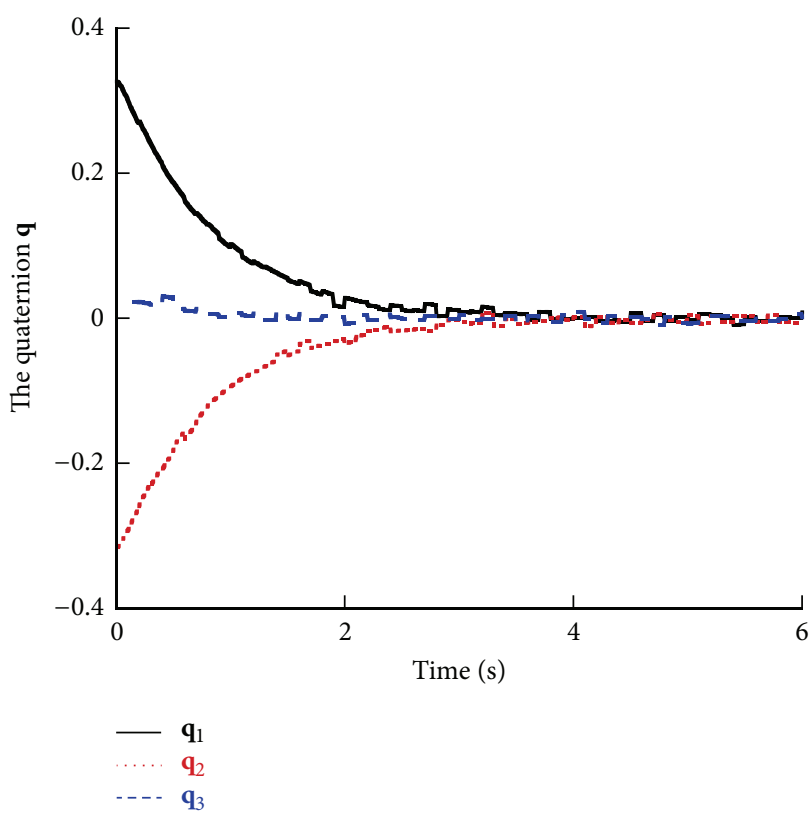

FIgURE 3: The response of the unit quaternion $\mathbf{q}$ in the absence of external disturbances.

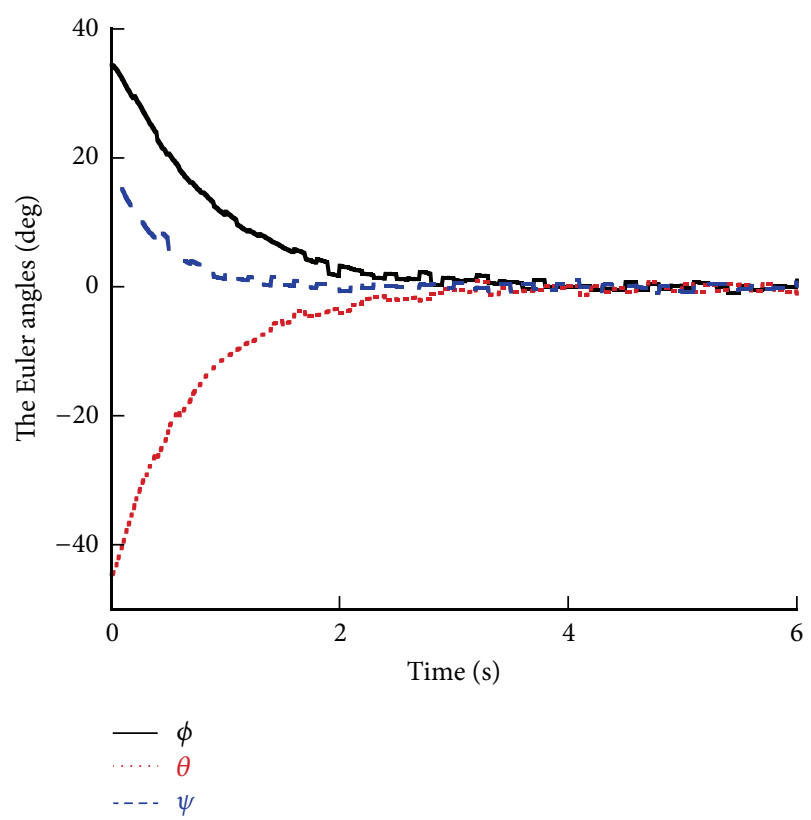

FIGURE 4: The response of Euler attitude angles in the absence of external disturbances.

(27), it leads to the control performance shown in Figures 3-5. It is observed from the response of the unit quaternion and the Euler attitude angles shown in Figures 3 and 4, respectively, that the attitude is successfully accomplished, while the resulted angular velocity is illustrated in Figure 5.

For a quadrotor flying outdoor, it is always under the effect of external disturbances such as induced by wind. Therefore, simulation is further carried out in the presence of external disturbances. Here, disturbances are introduced on 


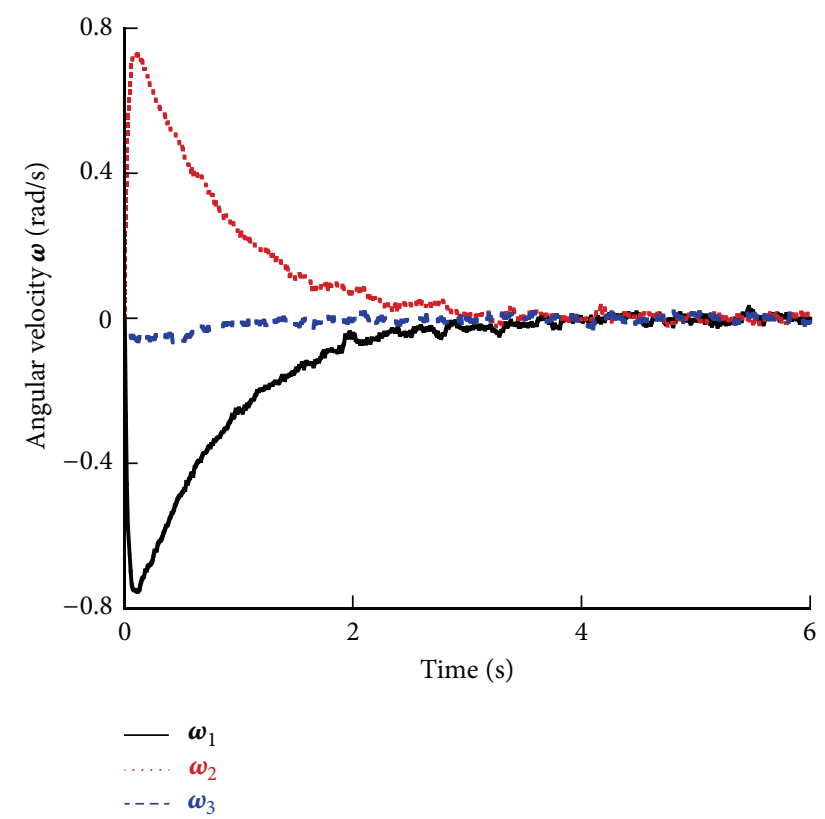

FIGURE 5: The angular velocity $\boldsymbol{\omega}$ in the absence of external disturbances.

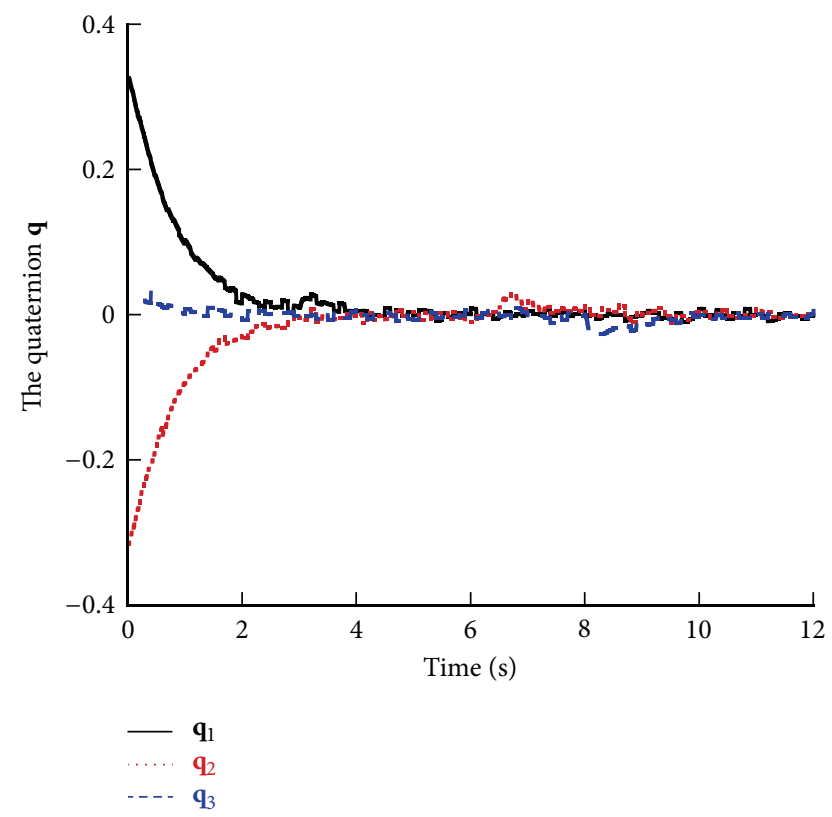

FIGURE 6: The response of the unit quaternion $\mathbf{q}$ in the presence of external disturbances.

the pitch, roll, and yaw at the 3th, 6.5th, and the 8th seconds, respectively. The attitude control performance obtained from the developed controller equation (27) is shown in Figures 68. From Figures 6-7, it is known that the disturbance torque has little effect on the performance of the attitude and that is because the controller equation (27) is able to guarantee the uniformly ultimately bounded stability of the closed-loop system in the presence of disturbance. It is seen in Figure 8 that although there exists some overshoot for the angular

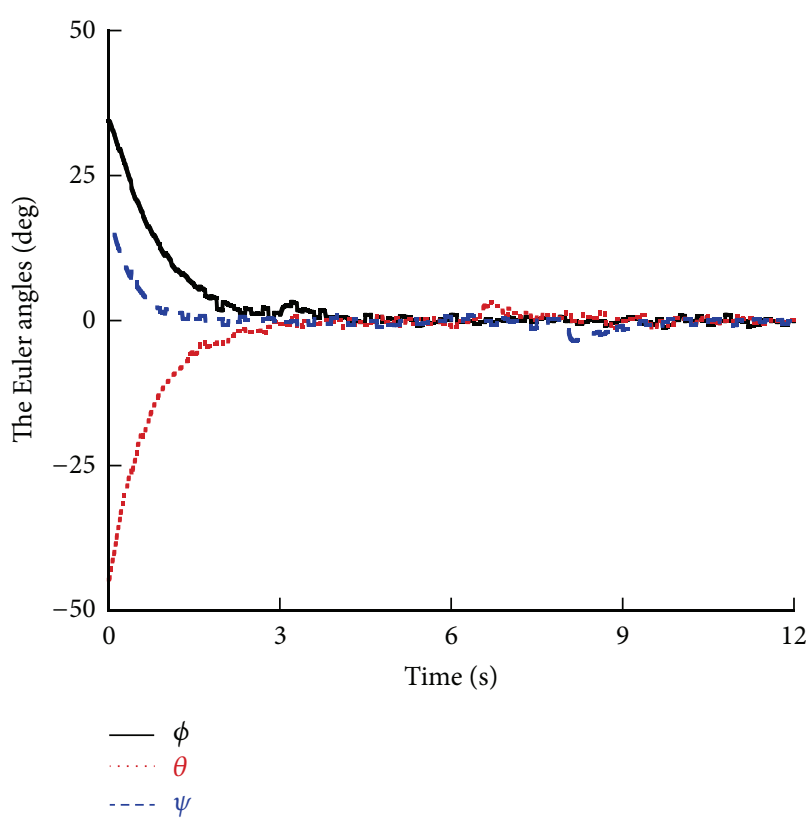

FIgURE 7: The response of Euler attitude angles in the presence of external disturbances.

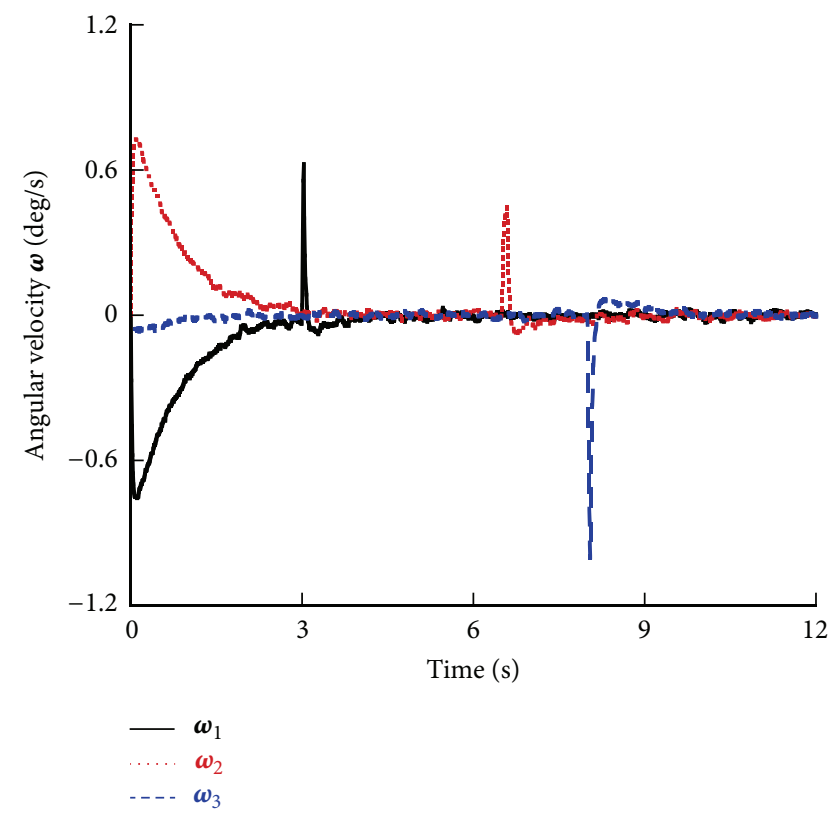

FIGURE 8: The angular velocity $\boldsymbol{\omega}$ in the presence of external disturbances.

velocity when disturbance is introduced to the quadrotor, the angular velocity can be stabilized soon.

\section{Conclusions}

In this work, the design of a quadrotor UAV attitude stabilization controller using quaternion feedback and integrator backstepping was presented. With appropriate choice of integrator backstepping variables, both equilibrium points in 
the closed-loop system are proved to be asymptotically stable. Furthermore, taking external disturbance torque into consideration, the attitude and the angular velocity of the quadrotor were governed to be uniformly ultimately bounded by the proposed controller. Simulations of a quadrotor UAV were also presented to illustrate the performance of the controller and that the attitude of the UAV was regulated to the closest equilibrium point. Future work will emphasize the position or path following control of the quadrotor even in the presence of external disturbance.

\section{Conflict of Interests}

The authors declare that there is no conflict of interests regarding the publication of this paper.

\section{Acknowledgments}

This present work was supported partially by National Natural Science Foundation of China (Project no. 61304149) and Natural Science Foundation of Liaoning, China (Project no. 2013020044). The authors highly appreciate the above financial supports.

\section{References}

[1] C. Shen, Z. J. Rong, and M. Dunlop, "Determining the full magnetic field gradient from two spacecraft measurements under special constraints," Journal of Geophysical Research A, vol. 117, no. 10, 2012.

[2] W. C. Tu, S. R. Elkington, X. L. Li, W. L. Liu, and J. Bonnell, "Quantifying radial diffusion coefficients of radiation belt electrons based on global MHD simulation and spacecraft measurements," Journal of Geophysical Research A, vol. 117, no. 10, 2012.

[3] Y. D. Song and W. C. Cai, "New intermediate quaternion based control of spacecraft: part I-almost global attitude tracking," International Journal of Innovative Computing Information and Control, vol. 8, no. 10, pp. 7307-7319, 2012.

[4] G. M. Li and L. D. Liu, "Coordinated multiple spacecraft attitude control with communication time delays and uncertainties," Chinese Journal of Aeronautics, vol. 25, no. 5, pp. 698-708, 2012.

[5] F. Pantellini, S. Belheouane, N. Meyer-Vernet, and A. Zaslavsky, "Nano dust impacts on spacecraft and boom antenna charging," Astrophysics and Space Science, vol. 341, no. 2, pp. 309-314, 2012.

[6] X. Y. Gao, K. L. Teo, and G. R. Duan, "Robust $H_{\infty}$ control of spacecraft rendezvous on elliptical orbit," Journal of the Franklin Institute, vol. 349, no. 8, pp. 2515-2529, 2012.

[7] H. R. Williams, R. M. Ambrosi, N. P. Bannister, P. SamaraRatna, and J. Sykes, "A conceptual spacecraft radioisotope thermoelectric and heating unit (RTHU)," International Journal of Energy Research, vol. 36, no. 12, pp. 1192-1200, 2012.

[8] S. Laborde and A. Calvi, "Spacecraft base-sine vibration test data uncertainties investigation based on stochastic scatter approach," Mechanical Systems and Signal Processing, vol. 32, pp. 69-78, 2012.

[9] S. Yin, S. X. Ding, A. H. A. Sari, and H. Hao, "Data-driven monitoring for stochastic systems and its application on batch process," International Journal of Systems Science, vol. 44, no. 7, pp. 1366-1376, 2013.
[10] S. Yin, S. X. Ding, A. Haghani, H. Hao, and P. Zhang, "A comparison study of basic data-driven fault diagnosis and process monitoring methods on the benchmark Tennessee Eastman process," Journal of Process Control, vol. 22, no. 9, pp. 1567-1581, 2012.

[11] S. Yin, H. Luo, and S.-X. Ding, "Real-time implementation of fault-tolerant control systems with performance optimization," IEEE Transactions on Industrial Electronics, vol. 61, no. 5, pp. 2402-2411, 2013.

[12] S. Yin, G. Wang, and H. R. Karimi, "Data-driven design of robust fault detection system for wind turbines," Mechatronics, 2013.

[13] S. Yin, X. Yang, and H. R. Karimi, "Data-driven adaptive observer for fault diagnosis," Mathematical Problems in Engineering, vol. 2012, Article ID 832836, 21 pages, 2012.

[14] E. V. Morozov and A. V. Lopatin, "Design and analysis of the composite lattice frame of a spacecraft solar array," Composite Structures, vol. 94, no. 10, pp. 3106-3114, 2012.

[15] F. Nemec, O. Santolik, M. Parrot, and J. S. Pickett, "Magnetospheric line radiation event observed simultaneously on board Cluster 1, Cluster 2 and DEMETER spacecraft," Geophysical Research Letters, vol. 39, no. 18, 2012.

[16] L. Palin, C. Jacquey, J. A. Sauvaud et al., "Statistical analyzis of dipolarizations using spacecraft closely separated along $\mathrm{Z}$ in the near-Earth magnetotail," Journal of Geophysical Research A, vol. 117, no. 9, 2012.

[17] Y. Miyake, H. Usui, H. Kojima, and H. Nakashima, "Plasma particle simulations on stray photoelectron current flows around a spacecraft," Journal of Geophysical Research A, vol. 117, no. 9, 2012.

[18] M. J. Aschwanden, J. P. Wuelser, N. V. Nitta, J. R. Lemen, M. L. DeRosa, and A. Malanushenko, "First three-dimensional reconstructions of coronal loops with the STEREOA+B spacecraft: IV. Magnetic modeling with twisted force-free fields," The Astrophysical Journal, vol. 756, no. 2, article 124, 2012.

[19] R. E. Denton, B. U. Ö. Sonnerup, J. Birn et al., "Test of Shi et al. method to infer the magnetic reconnection geometry from spacecraft data: MHD simulation with guide field and antiparallel kinetic simulation," Journal of Geophysical Research A, vol. 117, no. 9, 2012.

[20] K. D. Bilimoria and E. R. Mueller, "Handling qualities of a capsule spacecraft during atmospheric entry," Journal of Spacecraft and Rockets, vol. 49, no. 5, pp. 935-943, 2012.

[21] H. Lu, Y.-H. Li, and C.-L. Zhu, "Robust synthesized control of electromechanical actuator for thrust vector system in spacecraft," Computers \& Mathematics with Applications, vol. 64, no. 5, pp. 699-708, 2012.

[22] S.-J. Dong, Y.-Z. Li, J. Wang, and J. Wang, "Fuzzy incremental control algorithm of loop heat pipe cooling system for spacecraft applications," Computers \& Mathematics with Applications, vol. 64, no. 5, pp. 877-886, 2012.

[23] A. T. Basilevsky, "Analysis of suspicious objects in TV panorama taken by Venera-9 spacecraft," Solar System Research, vol. 46, no. 5, pp. 374-378, 2012.

[24] C. Edwards and S. Spurgeon, Sliding Mode Control: Theory and Applications, Taylor \& Francis, Boca Raton, Fla, USA, 1998.

[25] K. B. McCoy, I. Derecho, T. Wong et al., "Insights into the extremotolerance of Acinetobacter radioresistens 50v1, a gram-negative bacterium isolated from the Mars Odyssey spacecraft," Astrobiology, vol. 12, no. 9, pp. 854-862, 2012. 
[26] K. Yong, S. Jo, and H. Bang, "A modified rodrigues parameterbased nonlinear observer design for spacecraft gyroscope parameters estimation," Transactions of the Japan Society For Aeronautical and Space Sciences, vol. 55, no. 5, pp. 313-320, 2012.

[27] J. X. Fan and D. Zhou, "Nonlinear attitude control of flexible spacecraft with scissored pairs of control moment gyros," Journal of Dynamic Systems Measurement and Control, vol. 134, no. 5, Article ID 054502, 5 pages, 2012.

[28] Y. C. Xu and Q. Chen, "Minimization of mass for heat exchanger networks in spacecrafts based on the entransy dissipation theory," International Journal of Heat and Mass Transfer, vol. 55, no. 19-20, pp. 5148-5156, 2012.

[29] H.-T. Cui and X.-J. Cheng, "Anti-unwinding attitude maneuver control of spacecraft considering bounded disturbance and input saturation," Science China Technological Sciences, vol. 55, no. 9, pp. 2518-2529, 2012.

[30] A.-M. Zou, K. D. Kumar, and Z.-G. Hou, "Attitude coordination control for a group of spacecraft without velocity measurements," IEEE Transactions on Control Systems Technology, vol. 20, no. 5, pp. 1160-1174, 2012.

[31] R. P. Praveen, M. H. Ravichandran, V. T. S. Achari, V. P. J. Raj, G. Madhu, and G. R. Bindu, "A novel slotless Halbach-array permanent-magnet brushless dc motor for spacecraft applications," IEEE Transactions on Industrial Electronics, vol. 59, no. 9, pp. 3553-3560, 2012.

[32] K. G. Klein, G. G. Howes, J. M. TenBarge, S. D. Bale, C. H. K. Chen, and C. S. Salem, "Using synthetic spacecraft data to interpret compressible fluctuations in solar wind turbulence," The Astrophysical Journal, vol. 755, no. 2, article 159, 2012.

[33] G. M. Chen, J. Y. Xu, W. B. Wang, J. H. Lei, and A. G. Burns, "A comparison of the effects of CIR- and CME-induced geomagnetic activity on thermospheric densities and spacecraft orbits: case studies," Journal of Geophysical Research A, vol. 117, no. $8,2012$.

[34] Z. Y. Tan, L. Dong, and F. L. Tang, "Monte Carlo calculations of characteristic quantities of low-energy electron irradiation to spacecraft dielectrics," Nuclear Instruments \& Methods in Physics Research Section B, vol. 285, pp. 86-93, 2012.

[35] H. K. Khalil, Nonlinear Systems, Prentice Hall, Upper Saddle River, NJ, USA, 3rd edition, 2002. 


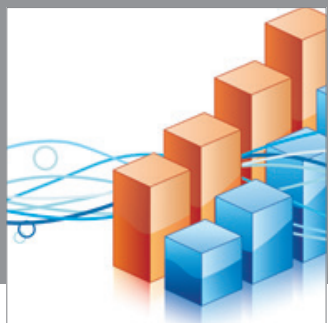

Advances in

Operations Research

mansans

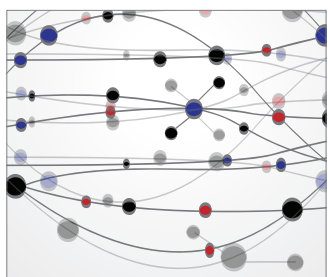

The Scientific World Journal
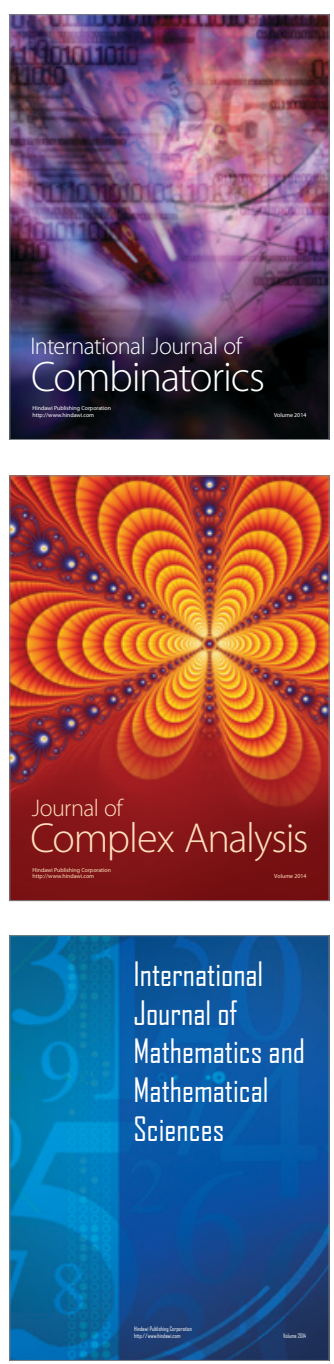
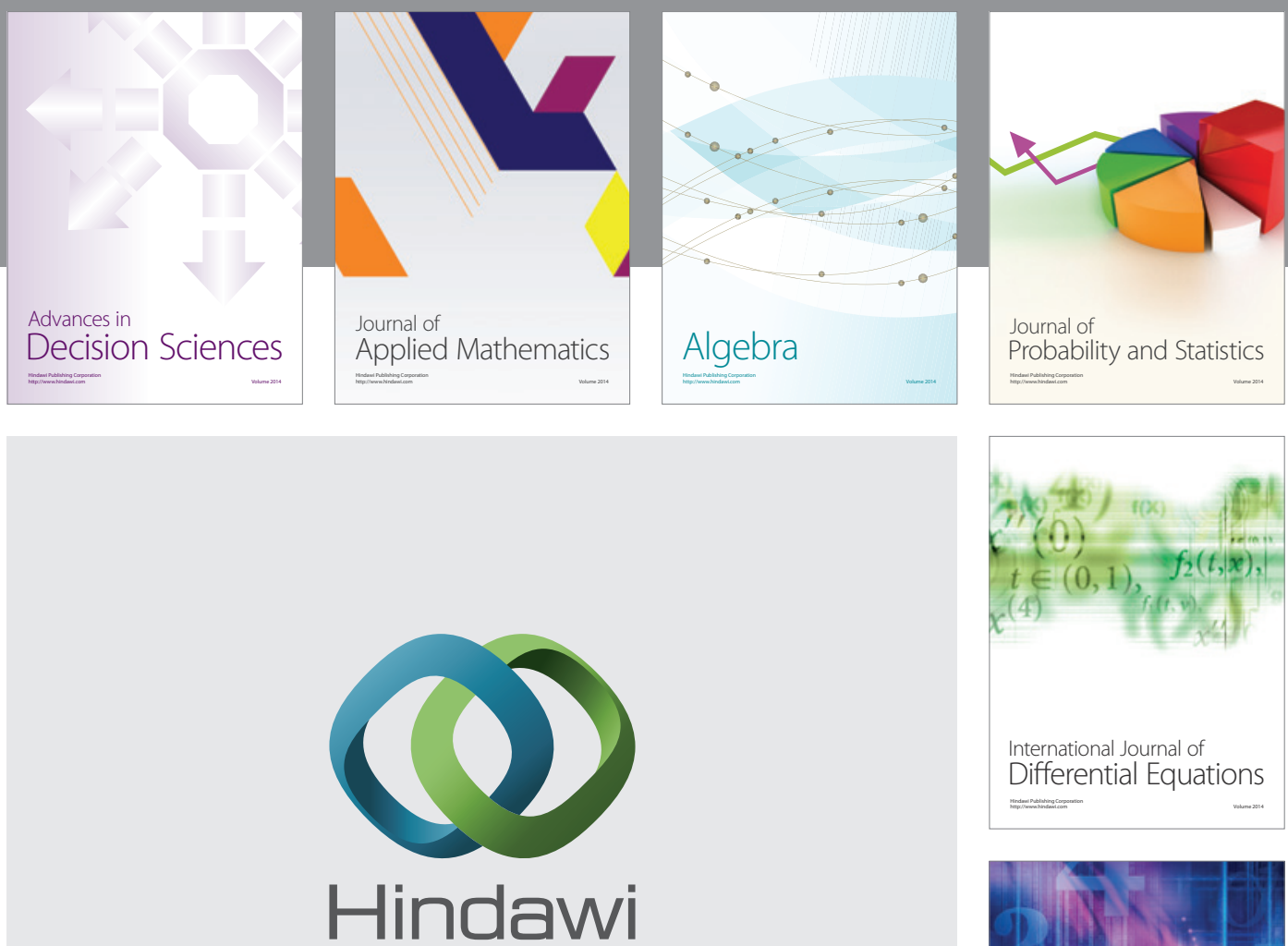

Submit your manuscripts at http://www.hindawi.com
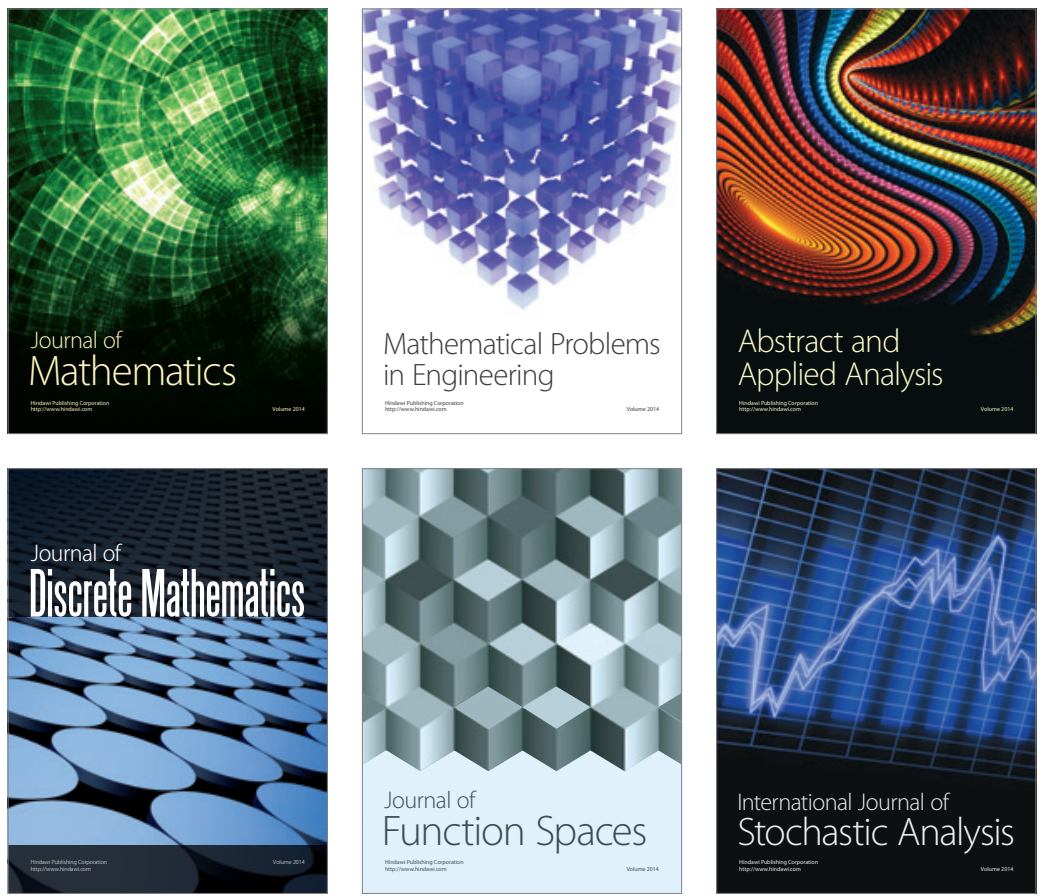

Journal of

Function Spaces

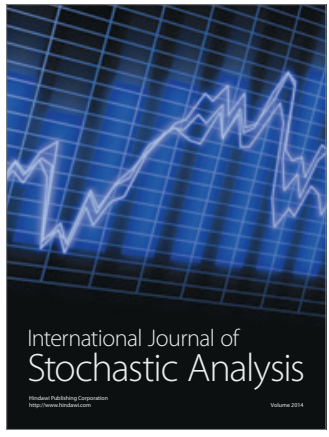

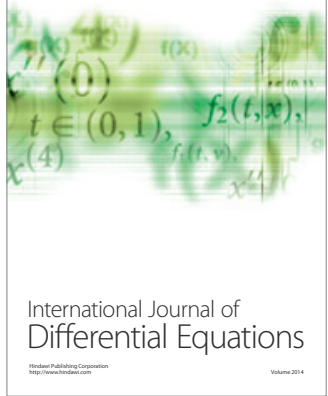
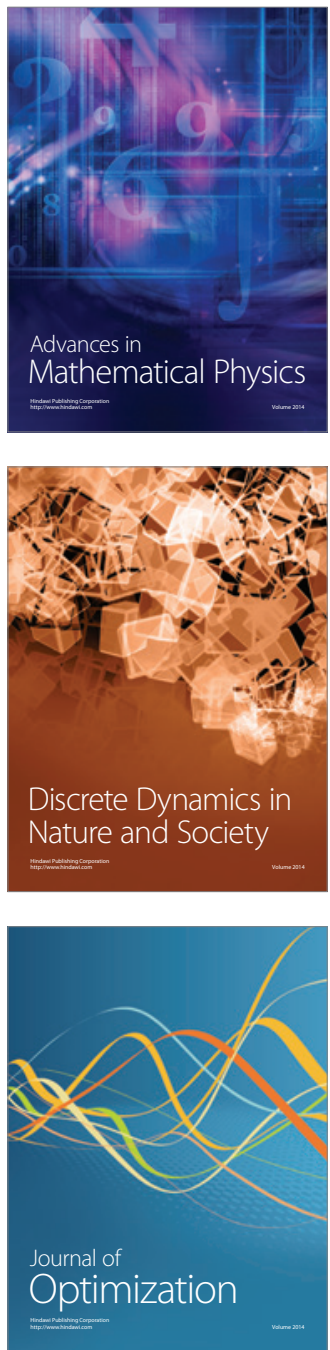